\title{
Testing lithium-ion battery with the internal reference electrode: an insight into the blocking effect
}

Zhengyu Chu, Xuning FENG, Yalun Li, Languang LU, Jianqiu Li, Xuebing Han, Minggao OUYANG, Boryann Liaw

October 2018

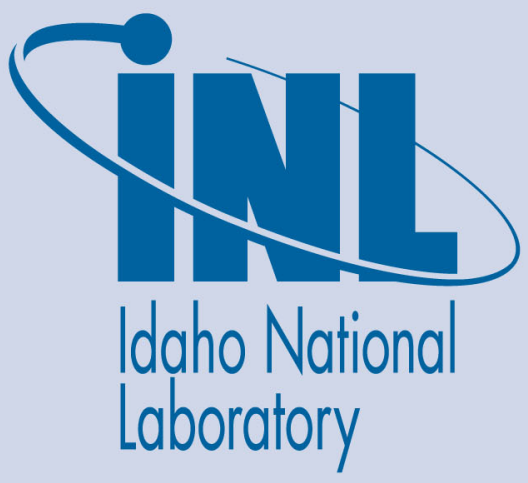

The INL is a U.S. Department of Energy National Laboratory operated by Battelle Energy Alliance 


\section{Testing lithium-ion battery with the internal reference electrode: an insight into the blocking effect}

Zhengyu Chu, Xuning FENG, Yalun Li, Languang LU, Jianqiu Li, Xuebing Han, Minggao OUYANG, Boryann Liaw

October 2018

Idaho National Laboratory Idaho Falls, Idaho 83415

http://www.inl.gov

Prepared for the U.S. Department of Energy Office of Nuclear Energy Under DOE Idaho Operations Office

Contract DE-AC07-05ID14517 


\section{.7.} 18

\title{
Testing lithium-ion battery with the internal reference electrode:
}

\section{an insight into the blocking effect}

\author{
Zhengyu Chu ${ }^{\mathrm{a}}$, Xuning FENG ${ }^{\mathrm{a}}$, Boryann Liaw ${ }^{\mathrm{b}}$, Yalun $\mathrm{Li}^{\mathrm{a}}$, Languang LU ${ }^{\mathrm{a}}$, Jianqiu Li ${ }^{\mathrm{a}}$, Xuebing \\ HAN $^{\mathrm{a}}$, Minggao OUYANG ${ }^{\mathrm{a}, *}$ \\ ${ }^{a}$ State Key Laboratory of Automotive Safety and Energy, Tsinghua University, Beijing 100084, China. \\ ${ }^{b}$ Energy Storage and Advanced Vehicles Department, Idaho National Laboratory, 2525 N. Fremont, Idaho \\ Falls, ID 83415, USA
}

\section{(1)}

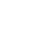

10

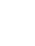

(1)

9 *Corresponding Author: ouymg@tsinghua.edu.cn

First author E-Mail: ouymg@tsinghua.edu.cn

Address: State Key Laboratory of Automotive Safety and Energy, Tsinghua University,

2 Beijing 100084, China

3 Tel.: (86)-10-62773437

4 Fax: (86)-10-62785708 


\section{Abstract:}

Long-term stability of reference electrode is a major issue in lithium-ion batteries. A composite $\mathrm{Ni}$-Li reference electrode $(\mathrm{RE})$ with a longer lifetime than traditional $\mathrm{Cu}$-wire $\mathrm{Li}$ reference electrodes is proposed in this study. However, the larger size of the Ni-Li RE creates a blocking effect when positioned internally in the battery. The blocking effect manifests as an abnormal feature in the measured electrode potential. A two-dimensional (2D) finite element model of the cell with an internal $\mathrm{RE}$ is constructed to investigate the mechanism of the blocking effect. The internal RE blocks the path of local current flow in the electrolyte, and decreases the electrochemical reaction rate of particles in the anode and cathode, leading to measurement errors.

Characteristic lengths of RE and applied currents were examined as parameters in the finite element model to determine their effect on the magnitude of error. A useful guide is provided for proper selection of size of RE and applied current in dynamic tests of lithium-ion batteries. 


\section{Introduction}

Lithium-ion batteries are widely used in consumer electronics, electric vehicles, and energy storage, because of their high energy and power density ${ }^{1,2}$. A RE is important in carrying out experimental tests and mechanistic investigations of lithium-ion batteries ${ }^{3-9}$. Electrode properties such as impedance or potential can be ascertained from information provided by a RE. This information enables some aging mechanisms and safety issues, such as solid electrolyte interphase (SEI) growth and such as internal short circuit ${ }^{10}$ caused by lithium deposition, to be identified. Although present commercial lithium-ion batteries do not have REs, most battery manufacturers are interested in monitoring the state of lithium plating with the help of a RE to improve battery safety and performance. Thus, there is a need for REs in battery research and industrial production.

Three categories of RE materials are used in lithium-ion batteries. The first, and most common, is lithium metal ${ }^{4,5,7-9,11-13}$, which is intrinsically compatible with the non-aqueous electrolyte of the battery, provides a stable value against which the electrode potential is measured. Although the electrode potential of lithium metal is $-3.04 \mathrm{~V}$ vs. the standard hydrogen electrode (SHE), it is customary to report potentials versus the $\mathrm{Li} / \mathrm{Li}^{+}$value. This is convenient for post-processing experimental data. Lithium metal is also readily and reproducibly produced. The second class of RE materials includes lithium compounds, such as $\mathrm{LiFePO}_{4}\left(3.4 \mathrm{~V}\right.$ vs. $\left.\mathrm{Li}^{2} \mathrm{Li}^{+}\right)$and $\mathrm{Li}_{4} \mathrm{Ti}_{5} \mathrm{O}_{12}(1.5$ $\mathrm{V}$ vs. $\mathrm{Li} / \mathrm{Li}^{+}$) etc. ${ }^{14}$, which have a known potential plateau. Lithium-containing RE must be oxidized to a specific state of charge (SOC) to provide a stable equilibrium potential. The third category of materials comprises binary lithium alloys, the most common of which is the Li-Sn alloy. ${ }^{3,15,16} \mathrm{Li}^{-\mathrm{Al}^{17}}, \mathrm{Li}-\mathrm{Au}^{18}$, and $\mathrm{Li}-\mathrm{Bi}^{6}$ alloys also have been recommended as $\mathrm{RE}$ materials for 
impedance and potential measurements. Lithium-alloy materials require a lithiation pretreatment to produce a stable reference potential. Because the lithium alloys typically exhibit more than one potential plateau, the process must be conducted carefully to produce the desired reference potential. A disadvantage of lithium compounds and alloys is reference potential drift due to lithium loss from the electrode. For this reason, we focus on lithium-metal RE materials.

Although REs are commonly used in potential and impedance measurements in lithium-ion batteries, some unsolved problems remain. For example, experimental distortions and artifacts are frequently observed. Jansen et al. investigated the effects of the RE size and placement and cell geometry on impedance measurements ${ }^{19}$. They concluded that a large internal RE can adversely affect impedance measurements and the misalignment of an external RE can cause errors in impedance measurements. Ender et al. evaluated the effects of geometric and electrical asymmetries using an external RE and found distortions in scaling factors and inductive artifacts in the impedance response ${ }^{20}$. Klink et al. demonstrated that geometrical asymmetry can be reduced by precise electrode alignment and use of a coaxial RE. ${ }^{21}$ Delacourt et al. proposed a novel three-electrode cell set-up, in which, holes in the working and counter electrodes for RE positioning minimized distortions in the impedance spectrum. ${ }^{22}$ Hoshi et al. suggested that the RE should be positioned externally between the positive and negative electrodes for optimum EIS measurements. ${ }^{23}$ Ender et al. examined point-like, wire, and mesh RE designs and demonstrated theoretically and experimentally that a mesh RE best achieves distortion-free EIS measurements. ${ }^{8}$, ${ }^{24}$ Although EIS measurements with a RE have been investigated extensively, only a few studies have examined the effects of RE placement and design in dynamic tests of lithium-ion batteries, which are important in investigating the fast charge ${ }^{11,25}$ and aging mechanism. ${ }^{4,26,27}$. Klett et al. 
compared positive and negative electrode potentials measured simultaneously with internal and external Res during a $10 \mathrm{~s}, 3 \mathrm{C}$ pulse charge, and found that the internal RE is more accurate. ${ }^{16}$ However, poor long-term stability of REs is a major issue, particularly in investigations of capacity fade. Designs based on Li-plating onto a $\mathrm{Cu}$ wire exhibit potential drift shortly after production, because the small amount of lithium is easily dissolved or consumed by SEI growth. Lithium alloys, which exhibit multiple potential plateaus, presumably suffer from potential drift when the intercalation state is unexpectedly altered. ${ }^{15}$ This also occurs with lithium compounds. Therefore, lithium metal remains the most suitable RE material for lithium-ion batteries, despite its possible depletion in the electrolyte.

In summary, an internal lithium RE, which provides more accurate electrode potential measurement, is preferred for dynamic testing, although a small size is needed to reduce blocking effects. However, the amount of active lithium must be as large as possible to extend electrode lifetime. The trade-off between measurement accuracy and lifetime is a limiting factor in the application of $\mathrm{Cu}$-wire based lithium REs.

In this study, a new RE design with a greater active lithium loading is proposed and compared in terms of performance with the Cu-wire-Li RE. The blocking effect of an internally positioned RE also is investigated under dynamic conditions based on experimental measurements and a $2 \mathrm{D}$ electrochemical model. Quantitative analysis of the blocking effect provides a general guide for selecting the proper characteristic length of RE and range of applied current.

\section{Experiment and model description}

\subsection{Reference electrode setup}

Two reference electrode setups were compared to evaluate the effect of RE geometry on electrode 
potential measurements in dynamic battery tests.

Lithium metal deposited onto a $\mathrm{Cu}$ wire substrate was used for Setup 1. The diameter of the $\mathrm{Cu}$ wire was $25 \mu \mathrm{m}$, which approximates the dimension of the separator. One tip of a 2-cm-long $\mathrm{Cu}$ wire was immersed in dilute sulfuric acid and washed thoroughly with stirring in water and ethanol. This process produced a $\mathrm{Cu}$ wire with a polished tip ready for lithium deposit. The polished tip was inserted carefully into the jelly roll between positive and negative electrodes. The inserted portion of $\mathrm{Cu}$ wire was covered by a separator to avoid an internal short circuit. After assembly, a 20- $\mu \mathrm{A}$ direct current was passed between the $\mathrm{Cu}$ wire and positive electrode and $\mathrm{Cu}$ wire and negative electrode successively for $1 \mathrm{~h}$ to form a uniform lithium deposit. ${ }^{28}$ This completes production of the $\mathrm{Cu}$-wire-Li RE.

Lithium metal applied to a Ni-foam substrate was used for Setup 2. The larger size of the Ni foam (ca. 0.1-mm thick $\times 1-\mathrm{mm}$ wide) provided greater lithium loading. As described by Cui et al. ${ }^{29}$ and Zhang et al. ${ }^{30}$, a piece of Ni foam was immersed into molten lithium, which formed a composite $\mathrm{RE}$ by infusion of Li into the Ni host. One side of the RE was attached to a Ni current collector by ultrasonic welding.

Subsequent experiments demonstrated that RE reliability and lifetime were greater with Setup 2. Because lithium is consumed during use, a greater amount of lithium leads to a longer RE lifetime $^{6,28}$. The superior performance of Setup 2 is attributed to its greater lithium loading, which unavoidably introduces blocking effects due to RE size.

\subsection{Experiment}

Two commercial $\mathrm{LiNi}_{0.5} \mathrm{Mn}_{0.3} \mathrm{Co}_{0.2} \mathrm{O}_{2}(\mathrm{NCM}) /$ graphite batteries with nominal capacities of $40 \mathrm{Ah}$ for Cell A and $24 \mathrm{Ah}$ for Cell B were selected for experimentation. Set-up 1 was applied to Cell A 
and Set-up 2 to Cell B. Setup 1 was applied to Cell A. Setup 2 was applied to Cell B. Cells A and B were large enough to neglect the impact of the RE on the cell terminal voltage. However, there was a difference in the time of RE emplacement. The $\mathrm{Cu}$ wire in Setup 1 was inserted into the jelly roll before the cell was sealed with the help of the manufacturer. In Setup 2, Cell B was discharged to $2.5 \mathrm{~V}$ and transferred to a dry room with a dewpoint of $-70{ }^{\circ} \mathrm{C}$. The Al-plastic film of Cell B was carefully cut open and partially peeled away to leave enough space to separate the positive and negative electrodes and insert the Li-Ni RE, which was wrapped with an additional piece of separator to inhibit short-circuiting. The cell was resealed using a new Al-plastic film with the Li-Ni RE positioned between the positive and negative electrodes.

It is worth mentioning that one-terminal and two-terminal configurations were typically used for application of REs. Although there is no issue of inconsistency in the one-terminal configuration, there is an undesirable interaction with the measuring circuit when the anode and cathode are monitored simultaneously. The effect is suppressed in the two-terminal configuration, but either format is feasible for electrode potential measurements. In this study, we adopt the one-terminal configuration, because it is sufficient to investigate the blocking effect of the RE.

A series of dynamic tests were performed on Cells A and B after the REs were implanted to examine the impact of RE size on electrode potential measurements.

1) Capacity test: Cells were charged with a constant current-constant voltage (CC-CV) protocol until the upper cut-off voltage and cut-off current were reached. This was followed by a constant current (CC) discharge to a lower cut-off voltage. A rest period was observed between charging and discharging. Three cycles were conducted to evaluate the reproducibility of the voltage measurement. The voltage ranges of Cells $\mathrm{A}$ and $\mathrm{B}$ were $4.2-2.8 \mathrm{~V}$ and $4.2-2.5 \mathrm{~V}$, respectively. 
The currents applied for charging and discharging, as suggested by the manufacturers, were 1 and 1/3 C for Cells A and B, respectively. The cut-off current was preset at $0.05 \mathrm{C}$ for both cells.

2) C-rate test: Cells were charged at C-rates of $0.2,0.5$, and $1 \mathrm{C}$. The C-rates of discharge suggested by the manufacturers were 1 and $1 / 3 \mathrm{C}$ for Cells A and B, respectively. A $2 \mathrm{~h}$ rest period was applied between charging and discharging to allow the cells to regain equilibrium.

3) RE test: This test was designed to compare different ranges of SOC operation. Two discharge protocols with differently terminated SOCs were performed with Cell B. The cell was first discharged at a rate of $1 / 3 \mathrm{C}$ from 0.03 to 0 SOC followed by a $1 \mathrm{~h}$ rest period. The cell was then charged to $0.5 \mathrm{SOC}$ at a rate of $1 / 2 \mathrm{C}$ followed by a $3 \mathrm{~h}$ rest period. Finally, the cell was discharged at a rate of $1 / 3 \mathrm{C}$ from 0.5 to $0.2 \mathrm{SOC}$ followed by a $3 \mathrm{~h}$ rest period.

All tests were performed on an 8-channel Neware BTS 4000 test station. The electrode potential was measured with a 6 1/2-digit multimeter (Keithley 2000) with an input impedance of $>10 \mathrm{G} \Omega$. The high input resistance reduces the leakage current to $100 \mathrm{pA}$ or less, which improves measurement accuracy and minimizes consumption of the reference electrode. The digital multimeter was used in combination with a 10-channel scanner (Keithley 2000-SCAN), which enabled simultaneous measurement of the anode and cathode potentials. The positive input of the multimeter was connected to the current collector of the negative/positive electrode, and the negative input was connected to the current collector of the reference electrode.

\subsection{Finite element model (FEM)}

A 2D FEM was built to investigate battery behavior with an internal RE. Fig. 1 shows the schematic shows a schematic of the 2D FEM geometry for simulation. Characteristic widths and thicknesses are considered in the model, which neglects the deformation of the separator and 
electrode caused by implanting the RE.

The electrochemical model is configured to a 2D description of the cell dynamics. Apart from the presence of the RE, the model is based on the work of $\mathrm{J}$. Newman et al. ${ }^{31}$ Charge and mass conservations are formulated by different partial differential equations (PDE) in the solid and electrolyte phases with corresponding boundary and initial conditions and coupled with a kinetic equation to describe the relationship between the overpotential and the lithium exchange flux (i.e., a Butler-Volmer equation). The principal equations are introduced here without their boundary and initial conditions.

Mass conservation in solid phase is given by:

$$
\frac{\partial c_{\mathrm{s}}}{\partial t}=\frac{D_{\mathrm{s}}}{r^{2}} \frac{\partial}{\partial r}\left(r^{2} \frac{\partial c_{\mathrm{s}}}{\partial r}\right)
$$

where $D_{\mathrm{s}}$ is the solid phase diffusion coefficient. Mass conservation in the electrolyte phase is given by:

$$
\begin{gathered}
\frac{\partial}{\partial t} \varepsilon_{e}^{\mathrm{j}} c_{e}=\nabla\left(D_{e, \text { eff }}^{\mathrm{j}} \nabla c_{e}\right)+a_{s}\left(1-t_{+}\right) j, \mathrm{j}=\mathrm{n}, \mathrm{p} \\
\frac{\partial}{\partial t} \varepsilon_{e}^{\mathrm{s}} c_{e}=\nabla\left(D_{e, \text { eff }}^{s} \nabla c_{e}\right)
\end{gathered}
$$

where $\varepsilon_{e}$ is the volume fraction of electrolyte, and $D_{e, \text { eff }}$ is the effective electrolyte diffusion coefficient. Eq. (2) is used in the negative and positive electrode regions, while Eq. (3) is used in the separator region. The lithium exchange flux, $j$, equals zero in the separator region. Charge conservation in the solid phase is:

$$
\nabla\left(\sigma_{\mathrm{eff}} \nabla \phi\right)-a_{s} F j=0
$$


where $\sigma_{\text {eff }}$ is the effective electrical conductivity in the solid phase. Charge conservation in the electrolyte phase is:

$$
\nabla\left(\kappa_{\text {eff }} \nabla \phi_{\mathrm{e}}\right)+\nabla\left(\frac{2 R T\left(t_{+}^{0}-1\right) \kappa_{\text {eff }}}{F}\left(1+\frac{\partial \ln f_{ \pm}}{\partial \ln c_{e}}\right) \nabla \ln c_{\mathrm{e}}\right)+a_{s} F j=0
$$

where $\kappa_{\text {eff }}$ is the effective electrical conductivity in the electrolyte phase. The kinetic equation has the form:

$$
\begin{gathered}
j=k_{0} c_{e}^{1-\alpha}\left(c_{s, \max }-c_{s, e}\right)^{1-\alpha} c_{s, e}{ }^{\alpha}\left(\exp \left(\frac{(1-\alpha) F}{R T} \eta\right)-\exp \left(\frac{-\alpha F}{R T} \eta\right)\right) \\
\eta=\phi_{\mathrm{s}}-\phi_{\mathrm{e}}-U_{\text {eq }}-F R_{\mathrm{SEI}} j
\end{gathered}
$$

where $k_{0}$ is reaction rate constant. The overpotential, $\eta$, is defined in Eq. (7), where $U_{\text {eq }}$ is the equilibrium potential for the charge transfer reaction.

The rectangular RE is positioned between the two electrodes as shown in Fig. 2. Lithium exchange occurs only on the surface of the RE. Therefore, the charge conservation in the RE region is:

$$
\nabla\left(\sigma^{\mathrm{RE}} \nabla \phi_{\mathrm{s}}\right)=0
$$

where $\sigma^{\mathrm{RE}}$ denotes the electrical conductivity of the RE (lithium metal). The electrode potential measured versus the solid phase potential of the RE is given by:

$$
\phi_{s}^{\mathrm{RE}}=\phi_{e}+U_{\mathrm{eq}}^{\mathrm{RE}}
$$

where the equilibrium of the RE is equals zero. This equation is applied on a presentative point on the surface of the RE and simulated electrode potential is equal to the difference between the solid potential of the electrode and the RE. All the simulations were carried out in Comsol Multiphysics. 


\section{Results and Discussion}

\subsection{Abnormal feature(s) of the electrode potential}

To determine if overall battery performance is affected by the presence of an internal RE, cell capacities were compared with and without the RE. For Setup A, the cell capacity of 40.39 Ah without the RE changed to 39.89 Ah after the RE was implanted. For Setup B, the cell capacity was 25.10 and 25.01 Ah before and after RE implantation, respectively. The internal RE appears to have little impact on the battery performance. The anode potential in Setup A was always greater than $0 \mathrm{~V}$ during the C-rate test, whereas the anode potential in Setup B decreased to $0 \mathrm{~V}$ at 0.5 and 1 C. The electrode potential profiles in Setup B also exhibited an abnormal feature compared with Setup A. Fig. 3 illustrates the characteristics of the abnormal feature by comparing electrode potential profiles for Setups A and B during rest periods in the C-rate tests. For Setup A, a continuous decrease is observed in the cathode or anode potential during the rest period, which is attributed to a depolarization. However, this behavior is not observed with Setup B. The at-rest anode potential after cell discharge first decreases for ca. $1 \mathrm{~h}$, but is followed immediately by a curious increase. Similar behavior also occurs after a rapid initial decrease in the at-rest cathode potential following cell charging. These observations contrast distinctly with the anticipated depolarization behavior of an electrode according to the electrochemical battery model.

The abnormal change in voltage that appears reproducibly in the dynamic experiments is considered to be a genuine feature rather than an experimental artifact. The presence of the feature demonstrates that an internal RE produces pronounced differences in electrode potential behavior during dynamic tests of lithium-ion batteries. Differences in the excitation time and time constant are observed between the cathode and anode potential responses, which suggest that the feature is 
governed by electrode architecture and material properties. The defining characteristic of the abnormal feature is the anomalous increase in anode/cathode potential during the rest period following cell discharge/charge rather than a continuous decrease until an equilibrium state is reached. A schematic depiction that highlights the abnormal feature is presented in Fig. 4 (b) with the abnormal increase highlighted. Fig. 4 (a), displays the normal response for comparison.

\subsection{Model fitting}

Specifically designed RE dynamic experiments were conducted to examine the origin, characteristics, and mechanism of the abnormal feature. The anode potential was the first parameter investigated. A short-term discharge was conducted to see if operation time was a factor. Cell B was allowed to rest for $3 \mathrm{~h}$ to ensure full relaxation and then discharged as shown in Fig. 5 (a) with a current of $1 / 3 \mathrm{C}$ from an initial voltage of $3.265 \mathrm{~V}$ to a cut-off voltage of $2.500 \mathrm{~V}$ for $264 \mathrm{~s}$ followed by a rest period of $1 \mathrm{~h}$. The anode potential in Fig. 5 (b) increases initially from 0.387 to $0.501 \mathrm{~V}$ until the end of discharge and then decreases to $0.473 \mathrm{~V}$ over the next $60 \mathrm{~s}$ followed by an increase to $0.553 \mathrm{~V}$ at the end of the rest period. This confirms that the abnormal feature is present even after a short period of operation.

A second experiment was conducted to examine the effect of the SOC on the abnormal feature, because the cell had been discharged to 0 SOC in all previous experiments. Cell B was adjusted to 0.5 SOC by first discharging to $0 \mathrm{SOC}$ and then recharging at $1 \mathrm{C}$ for $30 \mathrm{~min}$ followed by a $3 \mathrm{~h}$ rest period. The cell was then discharged from 0.5 to $0.2 \mathrm{SOC}$ at $1 / 3 \mathrm{C}$ for $0.86 \mathrm{~h}$ followed by another $3 \mathrm{~h}$ rest period as shown in Fig. 6 . The anode potential increases from 0.147 to $0.219 \mathrm{~V}$ during discharge and then decreases to $0.207 \mathrm{~V}$ over $380 \mathrm{~s}$ before reversing and increasing slightly 
244 in the second experiment, wherein the potential increment is $0.002 \mathrm{~V}$ rather than $0.080 \mathrm{~V}$. This

245 difference may result from operation over different SOC ranges, which will be elaborated upon in

246 the next section.

\subsection{An explanation of the abnormal feature: blocking effect}

Very little discussion was found in the literature on the behavior we have observed, because

examined during rest periods. The origin of the abnormal feature is explored in this section. There are two likely sources of the difference in electrode potential behavior between Setup A and Setup

B.

The first possible cause is the RE itself, which suggests that its potential may deviate from the standard value. In this case, undesirable reactions may occur at the interface between the Li metal and electrolyte leading to SEI film formation. When the RE is immersed in the electrolyte, parasitic reactions between metal and electrolyte species occur immediately and form compact, insoluble products on the $\mathrm{RE}^{32}$. These reactions may alter the RE potential. However, the behavior of $\mathrm{Li}$ as a $\mathrm{RE}$ differs from that as an anode. Very little current is applied to the RE, because of the high input impedance of the voltmeter. Therefore, reactions between $\mathrm{Li}$ and the electrolyte should be minimal. The difference in time constant between the anode and cathode potential response indicates that the abnormal feature is not related to the RE, because this parameter should be similar in both cases.

263 A second possible cause is the blocking effect of the RE, which has not been considered in equilibrium EIS measurements. ${ }^{23,33}$ The proposed $2 \mathrm{D}$ electrochemical model provides insight into 
measurements.

Electrolyte concentration distribution around the RE during a 3200 s discharge followed by a 1800 s rest period is demonstrated in Fig. 7, where the anode, separator, and cathode are shown from left to right. Lithium ion transport from one electrode to another is blocked by the RE, which acts as a physical barrier and increases the electrolyte concentration on the anode side and decreases that on the cathode side. The maximum difference between the electrolyte concentration in the blocked area and that at the same $\mathrm{x}$-axis coordinate in the unblocked area, $\left(\Delta C_{\mathrm{e}}\right)_{\max }$, increases with time. The difference increases to about $1700 \mathrm{~mol} / \mathrm{m}^{3}$ at $3000 \mathrm{~s}$ and decreases to about $417 \mathrm{~mol} / \mathrm{m}^{3}$ at $5000 \mathrm{~s}$. The blocked area extends about $0.2 \mathrm{~mm}$ beyond the edge of the RE, which has a width of $0.4 \mathrm{~mm}$.

The average solid-phase composition of the anode during discharge is shown in Fig. 8. Anode particles in the blocked area have greater solid-phase concentration than those in the unblocked area. Consequently, lithium deintercalation of particles in the blocked area is driven primarily by the solid-phase concentration gradient, which impedes the flow of current between the two electrodes. However, unlike the electrolyte, the maximum difference in solid-phase concentration between the blocked and unblocked regions at the same x-axis displacement, $\left(\Delta C_{\mathrm{s}, \mathrm{avg}}\right)_{\max }$, does not increase. $\left(\Delta C_{\mathrm{e}}\right)_{\max }$ equals 3267,4169 , and $3755 \mathrm{~mol} / \mathrm{m}^{3}$, respectively, at $1000 \mathrm{~s} 2000 \mathrm{~s}$ and $3000 \mathrm{~s}$. During the rest period after $3200 \mathrm{~s},\left(\Delta C_{\mathrm{e}}\right)_{\max }$ decreases gradually with time to reach an equilibrium state, which indicates that the solid-phase lithium in the blocked area diffuses across the concentration gradient to the particles in the remaining area. This is not strictly a physical diffusional process, in which the electrolyte bridges between different particles, because the bulk solid-phase potential is nearly uniform over the entire region of the electrode. Thus, the abnormal 

remote from the RE.

The internally positioned $\mathrm{RE}$ not only blocks $\mathrm{Li}^{+}$transport in the electrolyte phase, but also inhibits the electrode reaction in the blocked area, which causes the active material there to exhibit hysteresis. This condition leads to an increase in the abnormal potential during the rest period. The anode of Cell B is partially blocked by the RE during discharge, when a solid-phase concentration gradient is established along the y-axis. The gradient accumulates during the discharging/charging process and diminishes during the subsequent rest period.

The overall current density at the anode is zero during rest, which means that the local current density is small. Thus, the local anode potential is described by:

$$
\phi_{\mathrm{s}}-\phi_{\mathrm{e}} \approx U_{\mathrm{eq}}\left(\frac{c_{s, \text { surf }}}{c_{s, \text { max }}}\right)
$$

298 Lithium diffusion from the blocked to the unblocked area produces a continual decrease in the solid-phase concentration in the blocked area leading to an increase in the equilibrium voltage, $U_{\text {eq }}$. The measured anode potential, $V_{\mathrm{a}}$, increases with increasing $U_{\mathrm{eq}}$, which is the origin of the abnormal feature. The 2D model replicates this process in good agreement with experimental results as shown in Fig. 5 and Fig. 6. The cell parameters used in the simulation are listed in Table 1. The different increases in anode potential observed for different final states of charge also are explained by this model. The differential curve of graphite is shown in Fig. 9. The slope is greater at low SOC than at high SOC, which indicates that the shift in $U_{\text {eq }}$ is greater at constant lithium diffusion.

\subsection{Parameter influence:}


accuracy, especially under dynamic CC charging or discharging conditions. The accuracy of the electrode potential data cannot be established simply by comparing the terminal voltage to the difference between the anode and cathode potentials. Thus, three different widths, thicknesses, and current rates were considered to examine their impact on the errors caused by the blocking effect. $\mu \mathrm{m}$, which is on the order of the diameter of the attached $\mathrm{Cu}$ wire. The maximum width is similar to the width of the Ni-Li RE.

\subsubsection{Width:}

To evaluate the effect of RE width on the measurement, a CC charge simulation was carried out for widths of 25,300 , and $800 \mu \mathrm{m}$. The simulation involves a CC charge starting from $0.8 \mathrm{SOC}$ to an upper cut-off voltage of $4.2 \mathrm{~V}$ followed by $1800 \mathrm{~s}$ rest period. The average solid-phase concentration and electrode potential are represented in Fig. 10. To obtain a more explicit electrode/separator interface. with increasing width. Fig. 10 (b) shows that the measured cathode potential decreases 
the measured and real cathode potential is 2,11 , and $27 \mathrm{mV}$ at widths of 25,300 , and $800 \mu \mathrm{m}$,

respectively. The difference in the solid concentration diminishes progressively during the rest period, as does the error in the measured cathode potential. The solid concentration at the anode is less in the blocked than in the unblocked area consistent with the hysteresis of intercalation. Likewise, the measured anode potential is less than its true value, which indicates that lithium deposition may be overestimated under some conditions during measurement. For example, if a battery with an $800-\mu \mathrm{m}$-wide RE is charged from $0.8 \mathrm{SOC}$ at $1 / 3 \mathrm{C}$, experimental data indicate that lithium deposition will occur within $1380 \mathrm{~s}$ as the measured anode potential reaches $0 \mathrm{~V}$ vs $\mathrm{Li} / \mathrm{Li}^{+}$, not as shown in Fig. $10(\mathrm{~d})$.

\subsubsection{Thickness}

Fig. 11 shows the cathode and anode potentials at various RE thicknesses using the same charging protocol as in section 3.4.1. The measured potential is less than the anticipated value for both the cathode and the anode at each thickness. When the RE thickness is $5 \mu \mathrm{m}$, the average maximum error of the anode and cathode potentials is $8 \mathrm{mV}$. The maximum error increases only by 4 and 7 $\mathrm{mV}$ at thicknesses of 20 and $100 \mu \mathrm{m}$, respectively. Thus, thickness has a less significant influence than width, which is the more important factor to be considered. Please note that there is a gap between the positive and negative electrodes where the RE is inserted, leading to deformation of the electrodes. Little difference of the positive and negative electrode potentials is seen between the gap is considered or not in simulation. For this reason, the deviation caused by gap is neglected for brevity. The deviation may become larger for larger width.

\subsubsection{C-rate}

Although the C-rate is not a RE parameter, its impact should be assessed. A greater C-rate generally requires a smaller RE. Fig. 12 shows the results of electrode potential measurements with and without a RE. The average maximum errors of the anode and cathode potentials become negligible 
at small C-rates as shown in Fig. 12. Thus, the electrode potential data are close to anticipated values under thermodynamic or quasi-equilibrium conditions, such as during EIS measurements or low $\mathrm{C}$-rate tests. However, the average maximum errors of the anode and cathode potentials become 11 and $37 \mathrm{mV}$ when the $\mathrm{C}$-rate increases to $1 / 3$ and $1 \mathrm{C}$, respectively. Therefore, data obtained from dynamic tests at high $\mathrm{C}$-rates must be scrutinized carefully to ensure their validity. 
Table 3 summarizes the average errors of measured electrode potentials produced by simulation with different charging and discharging parameters. At widths of 25 and $300 \mu \mathrm{m}$, the average error is less than $15 \mathrm{mV}$ under all conditions, which is an acceptable value. However, the average error at $800 \mu \mathrm{m}$ increases to $76.5 \mathrm{mV}$ during discharge, which invalidates these experimental data. Thickness dependent errors are less than $15 \mathrm{mV}$ in all cases except at a $100 \mu \mathrm{m}$ thickness during discharge. Average electrode potential errors during charging and discharging are relatively consistent at all C-rates. Errors are less than $15 \mathrm{mV}$ at C-rates below 1/3 C, although the error exceeds $15 \mathrm{mV}$ at $1 \mathrm{C}$. The foregoing results provide important principles for proper application of REs. Our findings suggest that a conductive, mesh-like material with a proper proportion of skeleton and pores is an ideal RE substrate that is capable of reducing the blocking effect while loading enough lithium for long-term use. The performance of a RE with this design is being investigated by our group.

\section{Conclusions}

A new RE design with improved stability and lifetime is proposed for use in lithium-ion batteries. The proposed Ni-Li composite RE can load more lithium than traditional $\mathrm{Cu}$-wire-Li REs. In addition, the molten lithium infusion technique strengthens the attachment between the $\mathrm{Ni}$ substrate and lithium metal and prolongs RE lifetime. More insights into battery behavior can be obtained by use of a Ni-Li composite reference electrode of appropriate size. The impact of Ni-Li RE dimensions on electrode potential measurement were investigated in dynamic tests and evaluated on the basis of experimental results and a 2D electrochemical FEM. Abnormal deviations in negative/positive electrode potentials were observed in dynamic tests during the rest period following discharging/charging and were interpreted in terms of blocking by the internal 
RE. The internal RE physically blocks the surrounding Li-ion flow during charging and discharging and slows the electrochemical reaction of particles in the blocked area. This behavior introduces hysteresis and produces errors in electrode potential measurements. The abnormality arising from the blocked effect is reproduced by the 2D FEM. Characteristic lengths, widths, and thicknesses of the internal RE were analyzed by the FEM to quantify the errors caused by the blocking effect. The influence of current rate during dynamic tests also was evaluated. The salient conclusions of our investigation are as follows:

1) An internal RE with a width of less than $0.3 \mathrm{~mm}$ is sufficient to ensure an electrode potential measurement error of less than $15 \mathrm{mV}$.

2) An internal RE with a thickness of less than $0.02 \mathrm{~mm}$ is sufficient to ensure an electrode potential measurement error of less than $15 \mathrm{mV}$.

3) When using a 0.3-mm-wide and 0.02-mm-thick internal RE, an applied current rate equal to or less than $1 / 3 \mathrm{C}$ will produce an electrode potential measurement error equal to or less than $15 \mathrm{mV}$. Although specific errors may vary for different battery geometries or electrochemical characteristics, the method described here is useful in analyzing the blocking effects of an internal RE.

\section{Acknowledgement}

This work is supported by the National Natural Science Foundation of China (Grant No. U1564205), Ministry of Science and Technology of China (Grant No. 2016YES0102200). Authors also appreciate Prof. He Xiangming, Prof. Ai Xinping and Prof. Huang Jun for their valuable suggestions and Amperex Technology Limited for the technical support. 


\begin{tabular}{|c|c|c|c|}
\hline Parameter & Anode & Separator & Cathode \\
\hline Thickness $L(\mathrm{~m})$ & $52 \times 10^{-6}$ & $25 \times 10^{-6}$ & $50.6 \times 10^{-6}$ \\
\hline Solid phase conductivity $\sigma_{\mathrm{s}}\left(\mathrm{S} \cdot \mathrm{m}^{-1}\right)$ & $100^{34}$ & & $100^{34}$ \\
\hline Solid phase porosity $\varepsilon_{\mathrm{s}}$ & $0.63^{\mathrm{b}}$ & & $0.51^{\mathrm{b}}$ \\
\hline $\begin{array}{l}\text { Electrolyte phase conductivity } \kappa \\
\left(\mathrm{S} \cdot \mathrm{m}^{-1}\right)\end{array}$ & $\begin{array}{r}\kappa=0.0911+1.9101 \times 10 \\
\quad-1.052 \times 10^{-6} c_{\mathrm{e}}^{2}+0.1\end{array}$ & $\begin{array}{l}{ }^{-3} c_{\mathrm{e}} \\
554 \times 10^{-9} c_{\mathrm{e}}^{3}\end{array}$ & \\
\hline Electrolyte phase porosity $\varepsilon_{\mathrm{e}}$ & $0.3^{\mathrm{b}}$ & $1^{\mathrm{b}}$ & $0.3^{\mathrm{b}}$ \\
\hline $\begin{array}{l}\text { Solid diffusion coefficient } D_{\mathrm{s}} \\
\left(\mathrm{m}^{2} \cdot \mathrm{s}^{-1}\right)\end{array}$ & $\begin{array}{l}2.5 \times 10^{-14}(1.5-x)^{3.535} \\
\text { where } x=S O C\end{array}$ & & $2 \times 10^{-1435}$ \\
\hline $\begin{array}{l}\text { Electrolyte diffusion coefficient } D_{\mathrm{e}} \\
\left(\mathrm{m}^{2} \cdot \mathrm{s}^{-1}\right)\end{array}$ & $1.5 \times 10^{-1035}$ & & \\
\hline Particle radius $R_{\mathrm{s}}(\mathrm{m})$ & $2 \times 10^{-636}$ & & $3 \times 10^{-6}$ a \\
\hline Charge transfer coefficient $\alpha$ & $0.5^{36}$ & & $0.5^{36}$ \\
\hline SEI film resistance $R_{\text {SEI }}\left(\Omega \cdot \mathrm{m}^{2}\right)$ & $0.01^{36}$ & & $0^{36}$ \\
\hline Reaction rate $k\left(\mathrm{~m} \cdot \mathrm{s}^{-1}\right)$ & $8 \times 10^{-12}$ a & & $5.3 \times 10^{-12 \mathrm{a}}$ \\
\hline $\begin{array}{l}\text { maximum theoretical } \\
\text { concentration } c_{\mathrm{s}, \max }\left(\mathrm{mol} \cdot \mathrm{m}^{-3}\right)\end{array}$ & $31363^{37}$ & & $51385^{37}$ \\
\hline Faraday constant $\mathrm{F}\left(\mathrm{C} \cdot \mathrm{mol}^{-1}\right)$ & 96485 & & \\
\hline Transference number $t_{+}^{0}$ & $0.363^{\mathrm{a}}$ & & \\
\hline $\begin{array}{l}\text { Initial electrolyte concentration } c_{\mathrm{e}, 0} \\
\left(\mathrm{~mol} \cdot \mathrm{m}^{-3}\right)\end{array}$ & $1200^{\mathrm{a}}$ & $1200^{\mathrm{a}}$ & $1200^{\mathrm{a}}$ \\
\hline
\end{tabular}



Table 2 An overview of the parameter sets applied in simulation for evaulation of the devation caused 410 by RE

\begin{tabular}{cccc}
\hline Parameters & Width $/ \mu \mathrm{m}$ & Thickness $/ \mu \mathrm{m}$ & C-rate $/ \mathrm{C}$ \\
\hline Width & 25 & 20 & $1 / 3$ \\
& 300 & 20 & $1 / 3$ \\
& 800 & 20 & $1 / 3$ \\
Thickness & 300 & 5 & $1 / 3$ \\
& 300 & 20 & $1 / 3$ \\
& 300 & 100 & $1 / 3$ \\
C-rate & 300 & 20 & $1 / 20$ \\
& 300 & 20 & $1 / 3$ \\
& 300 & 20 & 1 \\
\hline
\end{tabular}

411

412 
Table 3 An overview of the deviation caused by RE using different widths, thicknesses and C-rates

Errors / $\mathrm{mV} \quad$ Width $/ \mu \mathrm{m} \quad$ Thickness / $\mu \mathrm{m} \quad$ C-rate / C

\begin{tabular}{ccccccccccc} 
& & 25 & 300 & 800 & 5 & 20 & 100 & $1 / 20$ & $1 / 3$ & 1 \\
\hline CHA & Neg & 0.6 & 6.4 & 26.6 & 4.7 & 6.4 & 9.5 & 0.8 & 6 & 21.1 \\
& Pos & 1.1 & 5.9 & 16.2 & 4.4 & 5.9 & 6.6 & 0.7 & 5.5 & 18.8 \\
& Average & 0.85 & 6.15 & 21.4 & 4.55 & 6.15 & 8.05 & 0.75 & 5.75 & 19.95 \\
\multirow{2}{*}{ DCH } & Neg & 0.3 & 10.3 & 54.5 & 6.7 & 10.3 & 13.3 & 1.2 & 7.7 & 22.2 \\
& Pos & 6.2 & 12.5 & 98.5 & 11.9 & 12.5 & 35.8 & 1.3 & 8.1 & 24.5 \\
& Average & 3.25 & 11.4 & 76.5 & 9.3 & 11.4 & 24.55 & 1.25 & 7.9 & 23.35
\end{tabular}

414

415 

composite RE.

Fig. 2 Schematic illustration of the 2D model geometry: 3D view (left) and cross section (right) with a inserted Ni-Li composite RE (white rectangle area) between the electrodes of the cell sandwich. The $2 \mathrm{D}$ model is constructed on the cross section.

421

Fig. 3 Cathode (blue solid line) and anode (green solid line) potentials in the cells with (a) the RE of Set-up A and (b) the RE of Set-up B during the C-rate tests. Zoomed views of the potentials during the rest period are shown to highlight the abonormal relaxation potential.

Fig. 4 A comparison of the anode/cathode potential profile during the rest period following a discharge/charge : (a) a normal profile, (b) an abnormal profile.

Fig. 5 A comparison between the experimental data and model prediction for (a) cell voltage and (b) anode potential in the cell with the RE of Set-up B, during a discharge to 0 SOC, followed by a $1 \mathrm{~h}$ rest period.

Fig. 6 A comparison between the experimental data and model prediction for (a) cell voltage and (b) anode potential in the cell with the RE of Set-up B, during a discharge to 0.2 SOC, followed by a 3 $\mathrm{h}$ rest period.

Fig. 7 Evolution of distribution for the electrolyte concentration at the adjacent region to the RE during a 3200 s discharge at $0.5 \mathrm{C}$ rate, followed by a $1800 \mathrm{~s}$ rest period.

Fig. 8 Evolution of distribution for the average solid phase concentration at the adjacent region to the RE during the same 3200 s discharge at $0.5 \mathrm{C}$ rate and the following $1800 \mathrm{~s}$ rest period in Fig. 7. Fig. 9 Equilibrium potential of the graphite anode (top) and its differential potential (bottom).

Fig.10 (a) Debiased average solid concentration along y-axis at the cathode/separator interface, (b) cathode potential, (c) debiased average solid concentraon along y-axis at the anode/separator interface, and (d) anode potential during a CC charge at $1 / 3 \mathrm{C}$ to $4.2 \mathrm{~V}$ and a following $1800 \mathrm{~s}$ rest period for different widths of the RE.

Fig. 11 (a) Cathode potential and (b) anode potential during a CC charge at $1 / 3 \mathrm{C}$ to $4.2 \mathrm{~V}$ and a following 1800 s rest period for different thicknesses of the RE.

Fig. 12 A comparison for the deviation caused by the RE at various C-rates. (a) Cathode potential and (b) anode potential during a $1000 \mathrm{~s}$ CC charge at different C-rates from $0.5 \mathrm{SOC}$, followed by a 1000 s rest period. 
1. L. Lu, X. Han, J. Li, J. Hua and M. Ouyang, J. Power Sources, 226, 272 (2013).

450

2. B. Scrosati and J. Garche, J. Power Sources, 195, 2419 (2010).

451

3. A. N. Jansen, D. W. Dees, D. P. Abraham, K. Amine and G. L. Henriksen, J. Power Sources, 174, $452373(2007)$.

453

4. P. Liu, J. Wang, J. Hicks-Garner, E. Sherman, S. Soukiazian, M. Verbrugge, H. Tataria, J. Musser and P. Finamore, J. Electrochem. Soc., 157, A499 (2010).

455

5. C. Huang, S. Zhuang and F. Tu, J. Electrochem. Soc., 160, A376 (2012).

456

6. J. L. Gómez-Cámer and P. Novák, Electrochem. Commun., 34, 208 (2013).

457

7. J. R. Belt, D. M. Bernardi and V. Utgikar, J. Electrochem. Soc., 161, A1116 (2014).

458

8. J. Costard, M. Ender, M. Weiss and E. Ivers-Tiffée, J. Electrochem. Soc., 164, A80 (2017).

459

9. T. Waldmann, B.-I. Hogg, M. Kasper, S. Grolleau, C. G. Couceiro, K. Trad, B. P. Matadi and M. Wohlfahrt-Mehrens, J. Electrochem. Soc., 163, A1232 (2016).

461

10. X. Feng, X. He, L. Lu and M. Ouyang, J. Electrochem. Soc., 165, A155 (2018).

462

11. T. Waldmann, M. Kasper and M. Wohlfahrt-Mehrens, Electrochim. Acta, 178, 525 (2015).

463

12. M. Itagaki, K. Honda, Y. Hoshi and I. Shitanda, J. Electroanal. Chem., 737, 78 (2015).

464

13. E. Barsoukov, J. H. Kim, J. H. Kim, C. O. Yoon and H. Lee, Solid State Ionics, 116, 249 (1999).

465

466

14. F. La Mantia, C. D. Wessells, H. D. Deshazer and Y. Cui, Electrochem. Commun., 31, 141 (2013).

15. D. P. Abraham, S. D. Poppen, A. N. Jansen, J. Liu and D. W. Dees, Electrochim. Acta, 49, 4763 (2004).

16. M. Klett, J. A. Gilbert, S. E. Trask, B. J. Polzin, A. N. Jansen, D. W. Dees and D. P. Abraham, J. Electrochem. Soc., 163, A875 (2016).

17. H. Nara, D. Mukoyama, T. Yokoshima, T. Momma and T. Osaka, J. Electrochem. Soc., 163, A434 (2015).

18. K. Mitsuda, S. Hara and D. Takemura, Electrochemistry, 84, 861 (2016).

19. D. W. Dees, A. N. Jansen and D. P. Abraham, J. Power Sources, 174, 1001 (2007).

20. M. Ender, A. Weber and I.-T. Ellen, J. Electrochem. Soc., 159, A128 (2011).

21. S. Klink, E. Madej, E. Ventosa, A. Lindner, W. Schuhmann and F. La Mantia, Electrochem. Commun., 22, 120 (2012).

22. C. Delacourt, P. L. Ridgway, V. Srinivasan and V. Battaglia, J. Electrochem. Soc., 161, A1253 (2014).

23. Y. Hoshi, Y. Narita, K. Honda, T. Ohtaki, I. Shitanda and M. Itagaki, J. Power Sources, 288, 168 480 (2015).

481 24. M. Ender, J. Illig and E. Ivers-Tiffée, J. Electrochem. Soc., 164, A71 (2017).

482 25. Z. Chu, X. Feng, L. Lu, J. Li, X. Han and M. Ouyang, Appl. Energ, 204, 1240 (2017).

483 26. M. Dubarry, C. Truchot, M. Cugnet, B. Y. Liaw, K. Gering, S. Sazhin, D. Jamison and C. 484 Michelbacher, J. Power Sources, 196, 10328 (2011).

485 27. M. Dubarry, C. Truchot and B. Y. Liaw, J. Power Sources, 258, 408 (2014).

486 28. J. Zhou and P. H. L. Notten, J. Electrochem. Soc., 151, A2173 (2004).

487 29. Y. Liu, D. Lin, Z. Liang, J. Zhao, K. Yan and Y. Cui, Nat Commun, 7, 10992 (2016).

488 30. S.-S. Chi, Y. Liu, W.-L. Song, L.-Z. Fan and Q. Zhang, Adv. Funct. Mater., 27, 1700348 (2017).

489 31. M. Doyle, T. F. Fuller and J. Newman, J. Electrochem. Soc., 140, 1526 (1993).

490 32. X. B. Cheng, R. Zhang, C. Z. Zhao, F. Wei, J. G. Zhang and Q. Zhang, Advanced Science, 3 
491 (2016).

492 33. Z. Wang, Z. Li, J. Huang and J. Zhang, ECS Transactions, 75, 151 (2017).

493 34. M. Doyle and J. Newman, J. Power Sources, 54, 46 (1995).

494 35. W. Fang, O. J. Kwon and C. Y. Wang, International journal of energy research, 34, 107 (2010).

495 36. S. Santhanagopalan, Q. Guo, P. Ramadass and R. E. White, J. Power Sources, 156, 620 (2006).

496 37. S. Tippmann, D. Walper, L. Balboa, B. Spier and W. G. Bessler, J. Power Sources, 252, 305 497 (2014).

498 